\title{
WEIGHTED BOUNDEDNESS OF THE 2-FOLD PRODUCT OF HARDY-LITTLEWOOD MAXIMAL OPERATORS
}

\author{
MARÍA J. CARRO* AND EDUARD ROURE**
}

\begin{abstract}
We study new weighted estimates for the 2-fold product of Hardy-Littlewood maximal operators defined by $M^{\otimes}(f, g):=M f M g$. This operator appears very naturally in the theory of bilinear operators such as the bilinear Calderón-Zygmund operators, the bilinear Hardy-Littlewood maximal operator introduced by Calderón or in the study of pseudodifferential operators. To this end, we need to study Hölder's inequality for Lorentz spaces with change of measures

$$
\|f g\|_{L^{p, \infty}\left(w_{1}^{p / p_{1}} w_{2}^{p / p_{2}}\right)} \leq C\|f\|_{L^{p_{1}, \infty}\left(w_{1}\right)}\|g\|_{L^{p_{2}, \infty}\left(w_{2}\right)} .
$$

Unfortunately, we shall prove that this inequality does not hold, in general, and we shall have to consider a weaker version of it.
\end{abstract}

\section{InTRODUCTION AND MOTIVATION}

Let us consider the Hardy-Littlewood maximal operator $M$, defined for locally integrable functions on $\mathbb{R}^{n}$ by

$$
M f(x):=\sup _{Q \ni x} \frac{1}{|Q|} \int_{Q}|f(y)| d y
$$

where the supremum is taken over all cubes $Q \subseteq \mathbb{R}^{n}$ containing $x$. The boundedness of $M$ in weighted Lebesgue spaces $L^{p}(w)$ is well understood since 1972 when Muckenhoupt [17] proved that, for every $1<p<\infty$,

$$
M: L^{p}(w) \longrightarrow L^{p}(w) \quad \Longleftrightarrow w \in A_{p},
$$

where $w \in A_{p}$ if $w$ is a positive and locally integrable function (called weight) such that

$$
[w]_{A_{p}}:=\sup _{Q}\left(\frac{1}{|Q|} \int_{Q} w(x) d x\right)\left(\frac{1}{|Q|} \int_{Q} w(x)^{1-p^{\prime}} d x\right)^{p-1}<\infty .
$$

Moreover, in the context of weak type inequalities, if $1 \leq p<\infty$,

$$
M: L^{p}(w) \longrightarrow L^{p, \infty}(w) \quad \Longleftrightarrow w \in A_{p},
$$

where $w \in A_{1}$ if

$$
M w(x) \leq C w(x), \quad \text { a.e. } x,
$$

2010 Mathematics Subject Classification. 42B99, 46E30.

Key words and phrases. Hölder's inequality, Muckenhoupt weights.

The authors were supported by grants MTM2016-75196-P (MINECO / FEDER, UE) and 2014SGR289.

E-mail addresses: * carro@ub.edu, ${ }^{* *}$ eroure@ub.edu . 
and the infimum of all such constants $C$ in the above inequality is denoted by $[w]_{A_{1}}$. Also, in the context of restricted weak type inequalities the following result was proved in [7, 13]:

$$
M: L^{p, 1}(w) \longrightarrow L^{p, \infty}(w) \quad \Longleftrightarrow w \in A_{p}^{\mathcal{R}},
$$

where a weight $w \in A_{p}^{\mathcal{R}}$ if

$$
[w]_{A_{p}^{\mathcal{R}}}:=\sup _{Q} w(Q)^{1 / p} \frac{\left\|\chi_{Q} w^{-1}\right\|_{L^{p^{\prime}, \infty}(w)}}{|Q|}<\infty .
$$

For any measurable set $F$, we write $w(F)=\int_{F} w(x) d x$; if $w=1$, we simply write $|F|$. Moreover,

$$
\|M\|_{L^{p, 1}(w) \rightarrow L^{p, \infty}(w)} \lesssim[w]_{A_{p}^{\mathcal{R}} .}
$$

Our main goal in this paper is to study weighted estimates for the 2-fold product of HardyLittlewood maximal operators, defined for locally integrable functions $f$ and $g$ in the most simple way:

$$
M^{\otimes}(f, g)(x):=M f(x) M g(x) .
$$

Most of the results in this paper can be extended to the $k$-fold product of Hardy-Littlewood maximal operators

$$
M^{\otimes}\left(f_{1}, \ldots, f_{k}\right)(x):=M f_{1}(x) \cdots M f_{k}(x),
$$

but, for simplicity, we shall only present the case $k=2$.

This operator has been very useful to obtain weighted estimates for several types of multilinear operators, such as the following ones:

1) The bilinear Hardy-Littlewood maximal operator: it was introduced by A. Calderón in 1964 and it is defined by

$$
\mathcal{M}(f, g)(x):=\sup _{r>0} \frac{1}{|B(0, r)|} \int_{B(0, r)}|f(x-y)||g(x+y)| d y .
$$

Using Hölder's inequality, we have that

$$
\mathcal{M}(f, g) \lesssim M\left(f^{1 / \theta}\right)^{\theta} M\left(g^{1 /(1-\theta)}\right)^{1-\theta},
$$

for every $0<\theta<1$ and hence,

$$
\mathcal{M}: L^{p_{1}}\left(\mathbb{R}^{n}\right) \times L^{p_{2}}\left(\mathbb{R}^{n}\right) \longrightarrow L^{p}\left(\mathbb{R}^{n}\right),
$$

for every $\frac{1}{p}=\frac{1}{p_{1}}+\frac{1}{p_{2}}$ and $p>1$, and he conjectured that

$$
\mathcal{M}: L^{2}\left(\mathbb{R}^{n}\right) \times L^{2}\left(\mathbb{R}^{n}\right) \longrightarrow L^{1}\left(\mathbb{R}^{n}\right) .
$$

This conjecture was shown to be true by Lacey in 2000 (see [14]), proving the unexpected fact that (1.1) holds for $p_{1}>1, p_{2}>1$ and $p>2 / 3$. In the same way, weighted estimates for the easier operator $M^{\otimes}$ will imply weighted estimates for $\mathcal{M}$. In particular, using Hölder's inequality, one can immediately obtain that

$$
M^{\otimes}: L^{p_{1}}\left(w_{1}\right) \times L^{p_{2}}\left(w_{2}\right) \longrightarrow L^{p}\left(w_{1}^{p / p_{1}} w_{2}^{p / p_{2}}\right),
$$

for every $p_{1}, p_{2}>1, \frac{1}{p}=\frac{1}{p_{1}}+\frac{1}{p_{2}}, w_{1} \in A_{p_{1}}$ and $w_{2} \in A_{p_{2}}$. Consequently,

$$
\mathcal{M}: L^{p_{1}}\left(w_{1}\right) \times L^{p_{2}}\left(w_{2}\right) \longrightarrow L^{p}\left(w_{1}^{p / p_{1}} w_{2}^{p / p_{2}}\right),
$$


for every $p_{1}>1 / \theta, p_{2}>1 /(1-\theta)$ and $w_{1} \in A_{\theta p_{1}}, w_{2} \in A_{(1-\theta) p_{2}}$. It is worth mentioning that much more delicate weighted estimates for the bilinear Hilbert transform have been recently obtained in [8].

2) Let now $T$ be a bilinear Calderón-Zygmund operator; that is, for every $f, g \in C_{c}^{\infty}$,

$$
T(f, g)(x)=\int_{\mathbb{R}^{2 n}} f\left(y_{1}\right) g\left(y_{2}\right) K\left(x, y_{1}, y_{2}\right) d y_{1} d y_{2}, \quad \forall x \notin \operatorname{supp} f \cap \operatorname{supp} g,
$$

where $K$ is defined away from the diagonal $x=y_{1}=y_{2}$, satisfies the size estimate

$$
\left|K\left(y_{0}, y_{1}, y_{2}\right)\right| \lesssim \frac{1}{\left(\sum_{k, l}\left|y_{k}-y_{l}\right|\right)^{2 n}}
$$

and for some $\varepsilon>0$ it satisfies the regularity condition

$$
\left|K\left(y_{0}, y_{1}, y_{2}\right)-K\left(y_{0}, y_{1}^{\prime}, y_{2}\right)\right| \lesssim \frac{\left|y_{1}-y_{1}^{\prime}\right|^{\varepsilon}}{\left(\sum_{k, l}\left|y_{k}-y_{l}\right|\right)^{2 n+\varepsilon}}
$$

and similarly for $\left|K\left(y_{0}, y_{1}, y_{2}\right)-K\left(y_{0}, y_{1}, y_{2}^{\prime}\right)\right|$. Let $T^{*}$ be its maximal truncated operator, defined by

$$
T^{*}(f, g)(x)=\sup _{\delta>0}\left|\int_{\left|x-y_{1}\right|^{2}+\left|x-y_{2}\right|^{2}>\delta^{2}} f\left(y_{1}\right) g\left(y_{2}\right) K\left(x, y_{1}, y_{2}\right) d y_{1} d y_{2}\right| .
$$

Then, L. Grafakos and R. H. Torres proved in [12] the following bilinear Cotlar's inequality: for every $\eta>0$, there exists a finite constant $C_{\eta}$ such that for every $(f, g) \in L^{p_{1}} \times L^{p_{2}}$, with $1 \leq p_{1}, p_{2}<\infty$, the following holds for all $x \in \mathbb{R}^{n}$ :

$$
T^{*}(f, g)(x) \leq C_{\eta}\left(M\left(|T(f, g)|^{\eta}\right)(x)^{1 / \eta}+M^{\otimes}(f, g)(x)\right) .
$$

As a consequence, one can deduce that

$$
T^{*}: L^{p_{1}}\left(w_{1}\right) \times L^{p_{2}}\left(w_{2}\right) \longrightarrow L^{p}\left(w_{1}^{p / p_{1}} w_{2}^{p / p_{2}}\right),
$$

with $w_{1} \in A_{p_{1}}$ and $w_{2} \in A_{p_{2}}$ by proving this estimate for the easier operators $T$ and $M^{\otimes}$. In this setting of bilinear Carderón-Zygmund integral operators, many other results have been proved where the role of the operator $M^{\otimes}$ is fundamental (see, for example, paper [16] where a good-lambda estimate for a maximal CZ operator with kernel satisfying a Dini condition is proved).

Concerning weighted bounds for $M^{\otimes}$, the above easy exercise (1.2) becomes an open question when we want to characterize the weights for which

$$
M^{\otimes}: L^{p_{1}, 1}\left(w_{1}\right) \times L^{p_{2}, 1}\left(w_{2}\right) \longrightarrow L^{p, \infty}\left(w_{1}^{p / p_{1}} w_{2}^{p / p_{2}}\right),
$$

and this is the question we want to address in this paper. In fact, the motivation comes from the recent restricted weak type Rubio de Francia extrapolation theory (see [2], 3]) where it has been proved that if an operator

$$
T: L^{p, 1}(w) \longrightarrow L^{p, \infty}(w),
$$

for every $w \in A_{p}^{\mathcal{R}}$, then endpoint $(1,1)$ estimates hold for characteristic functions; that is

$$
\left\|T \chi_{E}\right\|_{L^{1, \infty}(u)} \lesssim u(E), \quad \forall u \in A_{1},
$$


contrary to what happens with the classical Rubio de Francia theory. In this context, it has been proved in [4] that the operator $M^{\otimes}$ plays in the multilinear extrapolation theory of Rubio de Francia (see [11]) the same role that the classical Hardy-Littlewood maximal operator plays in the linear case of this theory. Therefore, the complete characterization of the weights $w_{1}$ and $w_{2}$ satisfying (1.3) becomes a fundamental and interesting question.

Obviously, if Hölder's inequality for Lorentz spaces with change of measures holds, as it happens with the $L^{p}$ spaces,

$$
\|f g\|_{L^{p, \infty}\left(w_{1}^{p / p_{1}} w_{2}^{p / p_{2}}\right)} \leq C\|f\|_{L^{p_{1}, \infty}\left(w_{1}\right)}\|g\|_{L^{p_{2}, \infty}\left(w_{2}\right)},
$$

and then, for every $w_{j} \in A_{p_{j}}^{\mathcal{R}}$,

$$
\left\|M^{\otimes}(f, g)\right\|_{L^{p, \infty}\left(w_{1}^{p / p_{1}} w_{2}^{p / p_{2}}\right)} \lesssim\|M f\|_{L^{p_{1}, \infty}\left(w_{1}\right)}\|M g\|_{L^{p_{2}, \infty}\left(w_{2}\right)} \lesssim\|f\|_{L^{p_{1}, 1}\left(w_{1}\right)}\|g\|_{L^{p_{2}, 1}\left(w_{2}\right)}
$$

as we expect. This is what happens in the particular case when all the weights are equal, $w:=w_{1}=w_{2}=w_{1}^{p / p_{1}} w_{2}^{p / p_{2}}$, since

$$
\|f g\|_{L^{p, \infty}(w)}=\sup _{t>0} t^{1 / p}(f g)_{w}^{*}(t) \lesssim \sup _{t>0} t^{1 / p}(f)_{w}^{*}(t)(g)_{w}^{*}(t) \leq\|f\|_{L^{p_{1}, \infty}(w)}\|g\|_{L^{p_{2}, \infty}(w)} .
$$

As a consequence:

Theorem 1.1. If $w \in A_{\min \left\{p_{1}, p_{2}\right\}}^{\mathcal{R}}$, then

$$
M^{\otimes}: L^{p_{1}, 1}(w) \times L^{p_{2}, 1}(w) \longrightarrow L^{p, \infty}(w) .
$$

However, we shall prove in Section 2 that (1.4) does not hold for general weights, and hence the complete solution to (1.3) remains open.

On the other hand, despite of the fact that (1.4) is not true, the following more intriguing and difficult result was proved in [15]: for every $w_{1}, w_{2} \in A_{1}$,

$$
M^{\otimes}: L^{1}\left(w_{1}\right) \times L^{1}\left(w_{2}\right) \longrightarrow L^{1 / 2, \infty}\left(w_{1}^{1 / 2} w_{2}^{1 / 2}\right) .
$$

Let us just mention here that this is the endpoint of the general case (1.3), which is surprising because usually endpoint estimates are harder to prove than estimates where, for example, all the spaces involved are Banach spaces.

The paper is organized as follows: we shall devote Section 2 to give a counterexample of (1.3) and prove a weaker version of Hölder's inequality for Lorentz spaces with change of measures which shall be fundamental for our main results in Section 3 concerning weighted boundedness of $M^{\otimes}$.

Before going on, let us recall the definition of the spaces which are going to be important for us (see [1]). Given $p>0$ and an arbitrary measure $\nu, L^{p, 1}(\nu)$ is the Lorentz space of measurable functions such that

$$
\|f\|_{L^{p, 1}(\nu)}:=p \int_{0}^{\infty} \lambda_{f}^{\nu}(y)^{1 / p} d y=\int_{0}^{\infty} f_{\nu}^{*}(t) t^{1 / p-1} d t<\infty
$$

and $L^{p, \infty}(\nu)$ is the Lorentz space of measurable functions such that

$$
\|f\|_{L^{p, \infty}(\nu)}:=\sup _{y>0} y \lambda_{f}^{\nu}(y)^{1 / p}=\sup _{t>0} t^{1 / p} f_{\nu}^{*}(t)<\infty
$$


where $f_{\nu}^{*}$ is the decreasing rearrangement of $f$ with respect to $\nu$, defined by

$$
f_{\nu}^{*}(t):=\inf \left\{y>0: \lambda_{f}^{\nu}(y) \leq t\right\}, \quad \lambda_{f}^{\nu}(t):=\nu(\{|f|>t\}) .
$$

As usual, we write $A \lesssim B$ if there exists a positive constant $C>0$, independent of $A$ and $B$, such that $A \leq C B$. If $A \lesssim B$ and $B \lesssim A$, then we write $A \approx B$.

Finally, unless indicated explicitly, we shall always assume that $1 \leq p_{1}, p_{2}<\infty$, although one can take $0<p_{i}<\infty$ whenever no conflict arises with the definition of the objects involved. Also, $w_{1}, w_{2}$ will denote weights on $\mathbb{R}^{n}$ and, by definition,

$$
\frac{1}{p}=\frac{1}{p_{1}}+\frac{1}{p_{2}} \quad \text { and } \quad w=w_{1}^{p / p_{1}} w_{2}^{p / p_{2}}
$$

\section{HÖLDER'S INEQUALITY FOR LORENTZ SPACES}

Let us start giving a counterexample that shows that (1.4) does not hold for general weights. Let us consider $n \geq 1,0<p_{1}, p_{2}<\infty$ and

$$
\begin{gathered}
f(x)=\frac{1}{|x|^{n / p_{1}}} \chi_{\{|x| \geq 1\}}, g(x)=|x|^{n / p_{1}} \chi_{\{|x| \geq 1\}}, \\
w_{1}(x)=1, w_{2}(x)=\frac{1}{|x|^{n\left(1+\frac{p_{2}}{p_{1}}\right)}} \chi_{\{|x| \geq 1\}}+\chi_{\{|x|<1\}} .
\end{gathered}
$$

Then $f g=\chi_{\{|x| \geq 1\}}$ and $w(x)=w_{2}(x)^{p / p_{2}}=\frac{1}{|x|^{n}} \chi_{\{|x| \geq 1\}}+\chi_{\{|x|<1\}}$, and hence,

$$
\|f g\|_{L^{p, \infty}(w)}^{p}=\int_{\{|x| \geq 1\}} \frac{1}{|x|^{n}} d x=+\infty
$$

while $\|f\|_{L^{p_{1}, \infty}\left(w_{1}\right)}=\|f\|_{L^{p_{1}, \infty}}<\infty$, and

$$
\|g\|_{L^{p_{2}, \infty}\left(w_{2}\right)} \leq \sup _{s>0} s\left(\int_{\left\{x \in \mathbb{R}^{n}:|x|^{\left.n / p_{1}>s\right\}}\right.} \frac{1}{|x|^{n\left(1+\frac{p_{2}}{p_{1}}\right)}} d x\right)^{1 / p_{2}}<\infty,
$$

and the result follows.

Due to this fact and in order to prove our main estimate for the operator $M^{\otimes}$, we need the following weaker versions of Hölder's inequality.

Lemma 2.1. Given a measurable set $E$ and a measurable function $g$,

$$
\left\|\chi_{E} g\right\|_{L^{p, \infty}(w)} \leq\left\|\chi_{E}\right\|_{L^{p_{1}, \infty}\left(w_{1}\right)}\|g\|_{L^{p_{2}, \infty}\left(w_{2}\right)} .
$$

Proof. If the right-hand side is infinite, then there is nothing to prove, so we may assume that $w_{1}(E)<\infty$ and $\|g\|_{L^{p_{2}, \infty}\left(w_{2}\right)}<\infty$. Now, for every $t>0$, we have that $\left\{\chi_{E}|g|>t\right\}=$ $E \cap\{|g|>t\}$ and hence, by Hölder's inequality,

$$
\begin{aligned}
t w\left(\left\{\chi_{E}|g|>t\right\}\right)^{1 / p} & \leq t w_{1}\left(\left\{\chi_{E}|g|>t\right\}\right)^{1 / p_{1}} w_{2}\left(\left\{\chi_{E}|g|>t\right\}\right)^{1 / p_{2}} \\
& \leq t w_{1}(E)^{1 / p_{1}} w_{2}(\{|g|>t\})^{1 / p_{2}},
\end{aligned}
$$

from which the result follows taking the supremum in $t>0$. 
Lemma 2.2. Given measurable functions $f$ and $g$, with $\|f\|_{\infty} \leq 1$, and $0<\delta<1$, we have that

$$
\|f g\|_{L^{p, \infty}(w)} \leq C(p, \delta)\left\|f^{\delta}\right\|_{L^{p_{1}, \infty}\left(w_{1}\right)}\|g\|_{L^{p_{2}, \infty}\left(w_{2}\right)} .
$$

Proof. Let $F$ be a measurable function with $\|F\|_{\infty} \leq 1$. Then, by Lemma 2.1, we have that

$$
\begin{aligned}
\sup _{0<t<1} t\left\|\chi_{\{|F|>t\}} g\right\|_{L^{p, \infty}(w)} & \leq \sup _{0<t<1} t\left\|\chi_{\{|F|>t\}}\right\|_{L^{p_{1}, \infty}\left(w_{1}\right)}\|g\|_{L^{p_{2}, \infty}\left(w_{2}\right)} \\
& \leq\|F\|_{L^{p_{1}, \infty}\left(w_{1}\right)}\|g\|_{L^{p_{2}, \infty}\left(w_{2}\right)} .
\end{aligned}
$$

Let $0<\delta<1$, set $F=f^{\delta}$ and fix $0<q<p$. By Kolmogorov's inequality (see 9] or [10, Ex. 1.1.12]),

$$
\|f g\|_{L^{p, \infty}(w)} \leq \sup _{0<w(A)<\infty}\left\|f g \chi_{A}\right\|_{L^{q}(w)} w(A)^{1 / p-1 / q},
$$

where the supremum is taken over all measurable sets $A$ with $0<w(A)<\infty$. For one of such sets $A$, we have that

$$
\begin{aligned}
\left\|f g \chi_{A}\right\|_{L^{q}(w)}^{q} & =\sum_{k<0} \int_{A \cap\left\{2^{k}<|f| \leq 2^{k+1}\right\}}|f g|^{q} w \leq 2^{q} \sum_{k<0} 2^{k q}\left\|\chi_{\left\{|f|>2^{k}\right\}} g \chi_{A}\right\|_{L^{q}(w)}^{q} \\
& \leq 2^{q} \sum_{k<0} 2^{k(1-\delta) q}\left(2^{\delta k}\left\|\chi_{\left\{|f|^{\delta}>2^{\delta k}\right\}} g \chi_{A}\right\|_{L^{q}(w)}\right)^{q} \\
& \leq \frac{2^{q}}{2^{(1-\delta) q}-1}\left(\sup _{0<t<1} t\left\|\chi_{\left\{|f|^{\delta}>t\right\}} g \chi_{A}\right\|_{L^{q}(w)}\right)^{q}
\end{aligned}
$$

and hence, applying Kolmogorov's inequality again,

$$
\begin{aligned}
& \sup _{0<w(A)<\infty}\left\|f g \chi_{A}\right\|_{L^{q}(w)} w(A)^{1 / p-1 / q} \\
& \leq 2\left(2^{(1-\delta) q}-1\right)^{-1 / q} \sup _{0<t<1} t \sup _{0<w(A)<\infty}\left\|\chi_{\left\{|f|^{\delta}>t\right\}} g \chi_{A}\right\|_{L^{q}(w)} w(A)^{1 / p-1 / q} \\
& \leq 2\left(2^{(1-\delta) q}-1\right)^{-1 / q}\left(\frac{p}{p-q}\right)^{1 / q} \sup _{0<t<1} t\left\|\chi_{\left\{|f|^{\delta}>t\right\}} g\right\|_{L^{p, \infty}(w)} \\
& \leq 2\left(2^{(1-\delta) q}-1\right)^{-1 / q}\left(\frac{p}{p-q}\right)^{1 / q}\left\|f^{\delta}\right\|_{L^{p_{1}, \infty}\left(w_{1}\right)}\|g\|_{L^{p_{2}, \infty}\left(w_{2}\right)} \\
& \leq 2\left(\frac{p}{\log 2(1-\delta) q(p-q)}\right)^{1 / q}\left\|f^{\delta}\right\|_{L^{p_{1}, \infty}\left(w_{1}\right)}\|g\|_{L^{p_{2}, \infty}\left(w_{2}\right)} .
\end{aligned}
$$

Hence, the theorem follows taking

$$
C(p, \delta):=\inf _{0<q<p} 2\left(\frac{p}{\log 2(1-\delta) q(p-q)}\right)^{1 / q}=2\left(\inf _{0<\theta<1}(\log 2(1-\delta) p \theta(1-\theta))^{-1 / \theta}\right)^{1 / p} .
$$

Observe that if $p>1$, then $C(p, \delta) \lesssim_{p} \frac{1}{1-\delta}$, and if $p \leq 1$, then $C(p, \delta) \lesssim_{p, \alpha} \frac{1}{(1-\delta)^{1+\alpha}}$, for every $\alpha>-1 / p^{\prime}$. 


\section{Main Results}

For our first lemma, recall that the class $A_{\infty}$ is simply defined by $A_{\infty}:=\bigcup_{p \geq 1} A_{p}$. See also the recent paper [5] for related results.

Lemma 3.1. If $w_{1}, w_{2} \in A_{\infty}$, then for every cube $Q$,

$$
w_{1}(Q)^{1 / p_{1}} w_{2}(Q)^{1 / p_{2}} \approx w(Q)^{1 / p} .
$$

Proof. Since $w_{1}, w_{2} \in A_{\infty}$, we have by Theorem 2.1 in [6] that $w_{i}^{p / p_{i}} \in R H_{p_{i} / p}, i=1$, 2 , and the result follows by Theorem 2.6 in the same paper.

Proposition 3.2. If $w_{1}, w_{2} \in A_{\infty}$, the following statements are equivalent:

(1) $w_{2} \in A_{p_{2}}^{\mathcal{R}}$.

(2) For every measurable set $E$ and every measurable function $g$,

$$
\left\|\chi_{E} M g\right\|_{L^{p, \infty}(w)} \lesssim w_{1}(E)^{1 / p_{1}}\|g\|_{L^{p_{2}, 1}\left(w_{2}\right)} .
$$

(3) For every cube $Q$ and every measurable function $g$,

$$
\left\|\chi_{Q} M g\right\|_{L^{p, \infty}(w)} \lesssim w_{1}(Q)^{1 / p_{1}}\|g\|_{L^{p_{2}, 1}\left(w_{2}\right)} .
$$

Proof. 2) follows from 1) applying Lemma 2.1, and it is clear that 3) follows from 2). Let us show that 3) implies 1). Fix a cube $Q$ and, using duality, choose a non-negative $g$ such that $\|g\|_{L^{p_{2}, 1}\left(w_{2}\right)} \leq 1$ and

$$
\left\|\chi_{Q} w_{2}^{-1}\right\|_{L^{p_{2}^{\prime}, \infty}\left(w_{2}\right)} \lesssim \int g\left(\chi_{Q} w_{2}^{-1}\right) w_{2}=\int_{Q} g .
$$

Since $\left.M g \geq\left(f_{Q} g\right) \chi_{Q}, 3\right)$ implies that

$$
\frac{w(Q)^{1 / p}}{|Q|}\left\|\chi_{Q} w_{2}^{-1}\right\|_{L^{p_{2}^{\prime}, \infty}\left(w_{2}\right)} \lesssim\left(f_{Q} g\right) w(Q)^{1 / p} \lesssim w_{1}(Q)^{1 / p_{1}} .
$$

Applying Lemma 3.1, we get that

$$
\frac{w_{2}(Q)^{1 / p_{2}}}{|Q|}\left\|\chi_{Q} w_{2}^{-1}\right\|_{L^{p_{2}^{\prime}, \infty}\left(w_{2}\right)} \lesssim 1
$$

and taking the supremum over all cubes $Q$, we get that $w_{2} \in A_{p_{2}}^{\mathcal{R}}$.

As a consequence we obtain our first main result.

Theorem 3.3. If $w_{1}, w_{2} \in A_{\infty}$ and (1.3) holds, then $w_{i} \in A_{p_{i}}^{\mathcal{R}}, i=1,2$.

Proof. Since $\chi_{E} \leq M \chi_{E}$, we have that

$$
\left\|\chi_{E} M f_{2}\right\|_{L^{p, \infty}(w)} \leq\left\|M \chi_{E} M f_{2}\right\|_{L^{p, \infty}(w)}=\left\|M^{\otimes}\left(\chi_{E}, f_{2}\right)\right\|_{L^{p, \infty}(w)} \lesssim w_{1}(E)^{1 / p_{1}}\left\|f_{2}\right\|_{L^{p_{2}, 1}\left(w_{2}\right)},
$$

and similarly,

$$
\left\|\chi_{E} M f_{1}\right\|_{L^{p, \infty}(w)} \leq\left\|M f_{1} M \chi_{E}\right\|_{L^{p, \infty}(w)}=\left\|M^{\otimes}\left(f_{1}, \chi_{E}\right)\right\|_{L^{p, \infty}(w)} \lesssim w_{2}(E)^{1 / p_{2}}\left\|f_{1}\right\|_{L^{p_{1}, 1}\left(w_{1}\right)} .
$$

The desired result follows from Proposition 3.2 . 
Observe that, in fact, the hypotheses that $w_{1}, w_{2} \in A_{\infty}$ can be replaced by (3.1).

We believe that the converse of the previous result holds. However, up to now, we need to assume some stronger condition in one of the weights; namely, either $w_{1} \in A_{p_{1}}$ or $w_{2} \in A_{p_{2}}$.

Theorem 3.4. Let $p_{1}>1$ and let $w_{1} \in A_{p_{1}}$ and $w_{2} \in A_{p_{2}}^{\mathcal{R}}$. Then, for every measurable set $E$ and every measurable function $g$,

$$
\left\|M^{\otimes}\left(\chi_{E}, g\right)\right\|_{L^{p, \infty}(w)} \lesssim\left\|\chi_{E}\right\|_{L^{p_{1}, 1}\left(w_{1}\right)}\|g\|_{L^{p_{2}, 1}\left(w_{2}\right)} .
$$

Proof. Since $w_{1} \in A_{p_{1}}$ and $p_{1}>1$, there exists $0<\delta<1$ such that $w_{1} \in A_{p_{1} \delta}$. Applying Lemma 2.2, we obtain that

$$
\begin{aligned}
\left\|M^{\otimes}\left(\chi_{E}, g\right)\right\|_{L^{p, \infty}(w)} & \lesssim\left\|M \chi_{E}\right\|_{L^{p_{1} \delta, \infty}\left(w_{1}\right)}^{\delta}\|M g\|_{L^{p_{2}, \infty}\left(w_{2}\right)} \\
& \lesssim\left\|\chi_{E}\right\|_{L^{p_{1} \delta, 1}\left(w_{1}\right)}^{\delta}\|g\|_{L^{p_{2}, 1}\left(w_{2}\right)} \approx\left\|\chi_{E}\right\|_{L^{p_{1}, 1}\left(w_{1}\right)}\|g\|_{L^{p_{2}, 1}\left(w_{2}\right)} .
\end{aligned}
$$

Observe that if $p>1$, this result can be extended to arbitrary measurable functions $f$ and $g$ by using that $L^{p, \infty}(w)$ is a Banach space.

Theorem 3.5. If $p, p_{1}>1, w_{1} \in A_{p_{1}}$ and $w_{2} \in A_{p_{2}}^{\mathcal{R}}\left(\right.$ or $p, p_{2}>1, w_{1} \in A_{p_{1}}^{\mathcal{R}}$ and $w_{2} \in A_{p_{2}}$ ), then

$$
M^{\otimes}: L^{p_{1}, 1}\left(w_{1}\right) \times L^{p_{2}, 1}\left(w_{2}\right) \longrightarrow L^{p, \infty}\left(w_{1}^{p / p_{1}} w_{2}^{p / p_{2}}\right) .
$$

Similar results can be proved for $A_{p}$ weights. We state them without proofs since these are completely analogous.

Proposition 3.6. If $w_{1}, w_{2} \in A_{\infty}$, then the following statements are equivalent:

(1) $w_{2} \in A_{p_{2}}$.

(2) For every measurable set $E$ and every measurable function $g$,

$$
\left\|\chi_{E} M g\right\|_{L^{p, \infty}(w)} \lesssim w_{1}(E)^{1 / p_{1}}\|g\|_{L^{p_{2}\left(w_{2}\right)}} .
$$

(3) For every cube $Q$ and every measurable function $g$,

$$
\left\|\chi_{Q} M g\right\|_{L^{p, \infty}(w)} \lesssim w_{1}(Q)^{1 / p_{1}}\|g\|_{L^{p_{2}\left(w_{2}\right)}} .
$$

Proposition 3.7. If $w_{1}, w_{2} \in A_{\infty}$ and $p_{2}>1$, then the following statements are equivalent:

(1) $w_{2} \in A_{p_{2}}$.

(2) For every measurable set $E$ and every measurable function $g$,

$$
\left\|\chi_{E} M g\right\|_{L^{p}(w)} \lesssim w_{1}(E)^{1 / p_{1}}\|g\|_{L^{p_{2}\left(w_{2}\right)}} .
$$

(3) For every cube $Q$ and every measurable function $g$,

$$
\left\|\chi_{Q} M g\right\|_{L^{p}(w)} \lesssim w_{1}(Q)^{1 / p_{1}}\|g\|_{L^{p_{2}\left(w_{2}\right)}} .
$$

Theorem 3.8. If $w_{1}, w_{2} \in A_{\infty}$, then

$$
M^{\otimes}: L^{p_{1}}\left(w_{1}\right) \times L^{p_{2}}\left(w_{2}\right) \longrightarrow L^{p, \infty}\left(w_{1}^{p / p_{1}} w_{2}^{p / p_{2}}\right),
$$

if and only if $w_{i} \in A_{p_{i}}, i=1,2$. And if $1<p_{1}, p_{2}<\infty$, this last condition is also equivalent to

$$
M^{\otimes}: L^{p_{1}}\left(w_{1}\right) \times L^{p_{2}}\left(w_{2}\right) \longrightarrow L^{p}\left(w_{1}^{p / p_{1}} w_{2}^{p / p_{2}}\right) .
$$




\section{REFERENCES}

[1] Bennett, C.; Sharpley, R. C.: Interpolation of Operators. Pure and Applied Mathematics, 129, Academic Press, Inc., Boston, MA, 1988.

[2] Carro, M. J.; Grafakos, L.; Soria, J.: Weighted weak-type $(1,1)$ estimates via Rubio de Francia extrapolation. J. Funct. Anal. 269 (2015), 1203-1233.

[3] Carro, M. J.; Soria, J.: Restricted weak-type Rubio de Francia extrapolation for $p>p_{0}$ with applications to exponential integrability estimates. Adv. Math. 290 (2016), 888-918.

[4] Carro, M.; Roure, E.: Restricted multilinear extrapolation theory. Preprint (2017).

[5] Cruz-Uribe, D.; Moen, K.: A multilinear reverse Hölder's inequality with applications to multilinear weighted norm inequalities. Preprint, arXiv:1701.07800 (2017).

[6] Cruz-Uribe, D.; Neugebauer, C. J.: The structure of the reverse Hölder classes. Trans. Amer. Math. Soc. 347 (1995), no. 8, 2941-2960.

[7] Chung, H. H.; Hunt, R.; Kurtz, D. S.: The Hardy-Littlewood maximal function on $L(p, q)$ spaces with weights. Indiana Univ. Math. J. 31 (1982), 109-120.

[8] Culiuc, A.; Di Plinio, F.; Ou, Y.: Domination of multilinear singular integrals by positive sparse forms. Preprint, arXiv:1603.05317v2 (2016).

[9] de Guzmán, M.: Real Variable Methods in Fourier Analysis. North-Holland Mathematics Studies, 46. Notas de Matemática [Mathematical Notes], 75. North-Holland Publishing Co., Amsterdam-New York, 1981.

[10] Grafakos, L.: Classical Fourier Analysis. Graduate Texts in Mathematics, 249, Springer, New York, 2014.

[11] Grafakos, L.; Martell, J. M.: Extrapolation of weighted norm inequalities for multivariable operators and applications. J. Geom. Anal. 14 (2004), no. 1, 19-46.

[12] Grafakos, L.; Torres, R. H.: Maximal operator and weighted norm inequalities for multilinear singular integrals. Indiana Univ. Math. J. 51 (2002), no. 5, 1261-1276.

[13] Kerman, R.; Torchinsky, A.: Integral inequalities with weights for the Hardy maximal function. Studia Math. 71 (1982), 277-284.

[14] Lacey, M.: The bilinear maximal functions map into $L^{p}$ for $2 / 3<p \leq 1$. Ann. of Math. 151 (2000), no. $1,35-57$.

[15] Lerner, A. K.; Ombrosi, S.; Pérez, C.; Torres, R. H.; Trujillo-González, R.: New maximal functions and multiple weights for the multilinear Calderón-Zygmund theory. Adv. Math. 220 (2009), no. 4, 1222-1264.

[16] Maldonado, D.; Naibo, V.: Weighted norm inequalities for paraproducts and bilinear pseudodifferential operators with mild regularity. J. Fourier Anal. Appl. 15 (2009), no. 2, 218-261.

[17] Muckenhoupt, B.: Weighted norm inequalities for the Hardy maximal function. Trans. Amer. Math. Soc. 165 (1972), 207-226.

Departament de Matemàtiques i Informàtica, Universitat de Barcelona, 08007 Barcelona, SPAIN.

E-mail address: carro@ub.edu

Departament de Matemàtiques i Informàtica, Universitat de Barcelona, 08007 Barcelona, SPAIN.

E-mail address: eroure@ub.edu 